\title{
Intravesical Coxsackievirus A21 Therapy for Non-Muscle Invasive Bladder Cancer
}

\author{
Edward Messing* \\ University of Rochester Medical Center, Rochester, NY, USA
}

Received 10 December 2019

Accepted 27 December 2019

Non-muscle invasive (NMI) urothelial cancer (UC) of the bladder has been responsive to intravesical therapy with live microorganisms (e.g. BCG) for well over 40 years [1] through mechanisms that induce direct cytotoxicity and more importantly, stimulation of the innate and adaptive immune systems. However, in view of repeated shortages of BCG [2] and because roughly 30-40\% of patients, particularly those with high risk disease, either do not respond to or cannot tolerate repetitive BCG instillations, $[3,4]$ there is need for other treatments of intermediate and high risk NMI UC. A series of articles by a British group using Coxsackievirus A21 intravesical therapy may provide a promising answer, at least based on preliminary mechanistic/translational data [5, 6]. It should be noted that the authors have not carried out needed studies to establish clinical efficacy, and there are reasons to believe that based on mechanistic studies, not all tumors will respond. However, this still represents a highly promising and welcome approach to treatment.

Annels and colleagues have demonstrated that Coxsackievirus A21, a naturally occurring RNA virus that causes the common cold, in vitro, in vivo in animal models, and when placed intravesically in patients can bind to ICAM-1 which is expressed only on the tumor cell's surface (and not on the

\footnotetext{
${ }^{*}$ Correspondence to: Edward M. Messing, MD, FACS, University of Rochester Medical Center, Rochester, NY, USA. Tel.: +1 585275 3345; E-mail: Messing@urmc.rochester.edu.
}

surfaces of non-malignant cells). Additionally, the virus' uptake is confined to UC cells. Besides being cytotoxic to cells which take the virus up, the resultant release of cytokines and immunoattractants by infected cells stimulates immune infiltrates. At least in animal (mouse) models, T4 helper cells are the immunoresponsive cells that primarily coordinate the host's attack on the tumor.

I CAM-1 expression appears to be necessary for Coxsackievirus A21's uptake by tumor cells and while this protein is not expressed on all UC cell lines, its expression in vitro can be upregulated by Mitomycin C (MMC) pre-treatment at concentrations far lower than those used as standard intravesical chemotherapy. At least in vitro, tumor cells not expressing I CAM-1 will not upregulate its expression with exposure to MMC. In a small sample of control non-treated patients with NMI UC, 43\% lacked ICAM-1 expression, making it unlikely that in these tumors Coxsackievirus A21 uptake will occur.

In a limited translational study in 15 patients with newly suspected solitary UC tumors, based upon cystoscopic appearance and positive cytology, received intravesical instillations of different doses of the virus +/- pretreatment with MMC at concentrations $<0.1 \%$ of that used clinically for intravesical therapy. Instillations were performed only once or on two consecutive days. Eight to 11 days later patients had transurethral resections (TURBTs) of their tumors. In these patients, whose tumors on TURBT histology were a mixture of low and high grade, mostly 
stage Ta lesions, it was not apparent that pretreatment with intravesical low dose MMC upregulated ICAM-1 expression.

However, once instilled, the virus replicated within cells, and the virus and immunostimulatory cytokines were excreted in urine. Also, markers of apoptosis and immunological cell death (e.g. high mobility group box 1 [HMBG1]) were expressed only in cancer cells. At most only two treatments were administered to any of the 15 patients, and one had a complete response. Treatments were well tolerated and viruses were not recovered from the systemic circulation in any patient.

So where does one go from here with this treatment?

As with any new therapy, many details must be worked out:

1. Can higher concentrations of MMC induce expression of ICAM-1?

2. How will this therapy eventually be utilized (e.g. repeated instillations one week apart after initial TURBT, as is used currently for intravesical chemo- and immunotherapy, or a different regimen)?

3. Will toxicity, which was not seen in this pilot study, occur with recurrent instillations?

Once these are addressed, phase II studies of efficacy should be performed. While still in preliminary stage, this work represents a promising new approach to treating NMI UC. Moreover, if effective, a major advantage of this treatment is that patients who will be unlikely to respond (those who's tumors do not express ICAM-1 initially) can be identified (possibly using immunocytology) and other treatments can be tried.

\section{CONFLICT OF INTEREST}

The author has no conflict of interest to report.

\section{REFERENCES}

[1] Morales A, Eidinger D, Bruce AW. Intracavitary Bacillus Calmette-Guerin in the treatment of superficial bladder tumors. J Urol. 1976;116(2):180-3.

[2] Messing EM. The BCG shortage. Bladder Cancer. 2017;3(3):227-8. doi: 10.3233/BLC-179018.

[3] Bassi P. BCG (Bacillus of Calmette Guerin) therapy of highrisk superficial bladder cancer. Surg Onc. 2002;11:77-83.

[4] Fuge O, Vasdev N, Allchorne P, et al. Immunotherapy for bladder cancer. Research and Reports in Urology. 2015;7:6579.

[5] Annels NE, Arif M, Simpson GR, et al. Oncolytic Immunotherapy for Bladder Cancer Using Coxsackie A21 Virus. Molecular Therapy: Oncolytics. 2018;9:1-12.

[6] Annels NE, Mansfield D, Arif M, et al. Viral targeting of nonmuscle invasive bladder cancer and priming of anti-tumour immunity following intravesical Coxsackievirus A21. July 4, 2019; doi:10.1158/1078-0432.CCR-18-4022 